Bull. Austral. Math. Soc.

VoL. 67 (2003) [503-510]

\title{
ON A BANACH SPACE PROPERTY OF TRUBNIKOV
}

\section{Simeon Reich AND Hong-Kun Xu}

Trubnikov's property $(U, \lambda, \alpha, \beta)$ is investigated. In particular, it is shown that property $(U, \lambda, \alpha, \alpha-1)$ with $\alpha>1$ is equivalent to $\alpha$-uniform smoothness. It is also shown that property $(U, 1, \alpha, 1)$ with $\alpha>1$ is equivalent to the space being a Hilbert space. The dual property $\left(U^{*}, \lambda, \alpha, \alpha-1\right)$ is also introduced and it is shown that a Banach space $X$ has $\left(U^{*}, \lambda, \alpha, \alpha-1\right)$ if and only if $X$ is $\alpha$-uniformly convex.

\section{InTRODUCTION}

Let $X$ be a real Banach space and let $\alpha, \lambda, \beta$ be real numbers with $\alpha \geqslant 1$. Trubnikov [6] introduced the concept of property $(U, \lambda, \alpha, \beta)$ as follows.

Definition 1.1: A Banach space $X$ has property $(U, \lambda, \alpha, \beta)$ if

$$
\|x+y\|^{\alpha}+\lambda\|x-y\|^{\alpha} \geqslant 2^{\beta}\left(\|x\|^{\alpha}+\|y\|^{\alpha}\right), \quad x, y \in X .
$$

Setting $u:=x+y$ and $v:=x-y$ in (1.1), we see that property $(U, \lambda, \alpha, \beta)$ is equivalent to the following inequality:

$$
\|x+y\|^{\alpha}+\|x-y\|^{\alpha} \leqslant 2^{\alpha-\beta}\left(\|x\|^{\alpha}+\lambda\|y\|^{\alpha}\right), \quad x, y \in X .
$$

Hence each Banach space has property $(U, 1, \alpha, 0)$; this is because

$$
\|u+v\|^{\alpha} \leqslant 2^{\alpha-1}\left(\|u\|^{\alpha}+\|v\|^{\alpha}\right), \quad u, v \in X,
$$

which implies that

$$
\|u+v\|^{\alpha}+\|u-v\|^{2} \leqslant 2^{\alpha}\left(\|u\|^{\alpha}+\|v\|^{\alpha}\right), \quad u, v \in X
$$

Note that we can always assume that $\lambda \geqslant 1$. We can also assume that $\beta \geqslant 0$. Indeed, the best possible value of $\beta$ such that (1.1) holds is given by

$$
2^{\beta}=\inf \left\{\frac{\|x+y\|^{\alpha}+\lambda\|x-y\|^{\alpha}}{\|x\|^{\alpha}+\|y\|^{\alpha}}:\|x\|^{\alpha}+\|y\|^{\alpha} \neq 0\right\} .
$$

\section{Received 11th November, 2002}

The first author was partially supported by the Fund for the Promotion of Research at the Technion and by the Technion VPR Fund. Both authors thank the referee for his/her comments and suggestions which improved the presentation of this paper.

Copyright Clearance Centre, Inc. Serial-fee code: 0004-9727/03 \$A2.00+0.00. 
Since $\lambda \geqslant 1$, we have from (1.1) that, for all $x, y \in X$,

$$
\|x+y\|^{\alpha}+\lambda\|x-y\|^{\alpha} \geqslant\|x+y\|^{\alpha}+\|x-y\|^{\alpha} \geqslant\|x\|^{\alpha}+\|y\|^{\alpha} .
$$

This implies $2^{\beta} \geqslant 1$; hence $\beta \geqslant 0$. Note that, given $\lambda$ and $\alpha$, a Banach space $X$ has property $(U, \lambda, \alpha, \beta)$ if and only if the infimum on the right-hand side of (1.4) is positive and $\beta$ is given by (1.4).

We now recall the following identity in a Hilbert space $H$ :

$$
\|t x+(1-t) y\|^{2}=t\|x\|^{2}+(1-t)\|y\|^{2}-t(1-t)\|x-y\|^{2}, \quad t \in[0,1], x, y \in H
$$

We also recall some inequalities in $l^{p}$ and $L^{p}$ spaces (see $[7,3]$ for more details).

1. If $2 \leqslant p<\infty$, then for all $x, y \in l^{p}$ (or $L^{p}$ ) and $t \in[0,1]$, there holds the inequality:

$$
\|t x+(1-t) y\|^{2} \geqslant t\|x\|^{2}+(1-t)\|y\|^{2}-t(1-t)(p-1)\|x-y\|^{2} .
$$

2. If $1<p<2$, then for all $x, y \in l^{p}$ (or $L^{p}$ ) and $t \in[0,1]$, there holds the inequality:

$$
\|t x+(1-t) y\|^{p} \geqslant t\|x\|^{p}+(1-t)\|y\|^{p}-c_{p} W_{p}(t)\|x-y\|^{p},
$$

where

$$
W_{p}(t)=t(1-t)^{p}+t^{p}(1-t) \quad \text { and } \quad c_{p}=\frac{1+s_{p}^{p-1}}{\left(1+s_{p}\right)^{p-1}}>1
$$

with $s_{p}$ being the unique solution of the equation

$$
(p-2) s^{p-1}+(p-1) s^{p-2}-1=0, \quad 0<s<1 .
$$

From (1.5)-(1.7) we can draw the following conclusion.

Proposition 1.2 .

(i) A Hilbert space $H$ has property $(U, 1,2,1)$.

(ii) If $2 \leqslant p<\infty$, then both $l^{p}$ and $L^{p}$ have property $(U, p-1,2,1)$.

(iii) If $1<p<2$, then both $l^{p}$ and $L^{p}$ have property $\left(U, c_{p}, p, p-1\right)$.

REMARK 1.3. For the spaces $L^{p}$ or $l^{p}$, Clarkson's inequalities [2, Theorem 2] also relate to property $(U, \lambda, \alpha, \beta)$. Indeed, Clarkson proves the following inequalities for $L^{p}$ or $l^{p}$ with $2 \leqslant p<\infty$ :

$$
2\left(\|x\|^{p}+\|y\|^{p}\right) \leqslant\|x+y\|^{p}+\|x-y\|^{p} \leqslant 2^{p-1}\left(\|x\|^{p}+\|y\|^{p}\right) .
$$


(For $1<p<2$ these inequalities hold in the reverse sense.) Hence $L^{p}$ or $l^{p}$ has $(U, 1, p, 1)$ for $2 \leqslant p<\infty$ and $(U, 1, p, p-1)$ for $1<p<2$. Note that $(U, 1, p, p-1)$ implies $\left(U, c_{p}, p, p-1\right)$ for $c_{p}>1$.

Trubnikov [6] introduced property $(U, \lambda, \alpha, \beta)$ to obtain the convergence rate of an iterative approximation method for nonlinear equations in Banach spaces. This direction has recently been pursued by several authors (see, for example, Schu [5] and references therein).

The purpose of this paper is to further study property $(U, \lambda, \alpha, \beta)$. In particular, we show that a Banach space $X$ has property $(U, \lambda, \alpha, \alpha-1)$ with $1<\alpha \leqslant 2$ if and only if $X$ is $\alpha$-uniformly smooth and that property $(U, 1, \alpha, 1)$ with $\alpha>1$ implies that $X$ is a Hilbert space. (For some related information on uniform convexity and uniform smoothness, the reader is referred to $[1,2,4]$.)

\section{2. $(U, \lambda, \alpha, \alpha-1)$ AND $\alpha$-UNIFORM SMOOTHNESS}

Recall that the modulus of smoothness of a Banach space $X$ is defined by

$$
\rho_{X}(\tau)=\sup \left\{\frac{1}{2}(\|x+\tau y\|+\|x-\tau y\|)-1:\|x\|=\|y\|=1\right\}, \quad \tau>0 .
$$

A Banach space $X$ is said to be uniformly smooth if

$$
\lim _{\tau \rightarrow 0^{+}} \frac{\rho_{X}(\tau)}{\tau}=0
$$

For a given number $q>1$, recall that $X$ is $q$-uniformly smooth if, for some constant $c>0$,

$$
\rho_{X}(\tau) \leqslant c \tau^{q}, \quad \tau>0 .
$$

It is known that $1<q \leqslant 2$. It is also known that a Hilbert space and $l^{p}$ (or $L^{p}$ ) for $2 \leqslant p<\infty$ are 2-uniformly smooth; while if $1<p<2, l^{p}$ (or $L^{p}$ ) is $p$-uniformly smooth (see [7]).

The following is an inequality characterisation of $q$-uniform smoothness (see $[7,3])$.

Proposition 2.1. Let $X$ be a Banach space and let $q \in(1,2]$ be a real number. Then $X$ is $q$-uniformly smooth if and only if there exists a constant $c>0$ with the property:

$$
\|t x+(1-t) y\|^{q} \geqslant t\|x\|^{q}+(1-t)\|y\|^{q}-c W_{q}(t)\|x-y\|^{q}
$$

for all $t \in[0,1]$ and $x, y \in X$, where $W_{q}(t)=t(1-t)^{q}+t^{q}(1-t)$.

Now we state and prove the main result of this paper.

Theorem 2.2. Let $X$ be a Banach space and let $\alpha>1$ be a real number. Then $X$ has property $(U, \lambda, \alpha, \alpha-1)$ for some $\lambda>0$ if and only of $X$ is $\alpha$-uniformly smooth. 
Proof: Assume that $X$ is $\alpha$-uniformly smooth. Substituting $t=1 / 2$ in (2.1) with $q$ replaced with $\alpha$, we obtain

$$
\|x+y\|^{\alpha} \geqslant 2^{\alpha-1}\left(\|x\|^{\alpha}+\|y\|^{\alpha}\right)-c\|x-y\|^{\alpha}, \quad x, y \in X .
$$

It follows that $X$ has property $(U, \lambda, \alpha, \alpha-1)$ with $\lambda=c$.

Conversely, assume that $X$ has property $(U, \lambda, \alpha, \alpha-1)$ for some $\lambda>0$ and $\alpha>1$. By (1.2), we have (note that $\alpha-\beta=1$ )

$$
\|x+\tau y\|^{\alpha}+\|x-\tau y\|^{\alpha} \leqslant 2\left(\|x\|^{\alpha}+\lambda \tau^{\alpha}\|y\|^{\alpha}\right), \quad x, y \in X, \tau>0 .
$$

In particular,

$$
\frac{1}{2}\left(\|x+\tau y\|^{\alpha}+\|x-\tau y\|^{\alpha}\right)-1 \leqslant \lambda \tau^{\alpha}, \quad x, y \in X,\|x\|=\|y\|=1, \tau>0 .
$$

Let

$$
D=\left\{(t, s) \in \mathbf{R}^{2}: 0 \leqslant t, s \leqslant 1+\tau, t+s \geqslant 2\right\},
$$

where we assume $\tau \in[0,1]$.

Claim. $\alpha[(t+s) / 2-1] \leqslant\left(t^{\alpha}+s^{\alpha}\right) / 2-1$ on $D$.

The proof of the Claim is elementary. We include it for completeness. Let

$$
h(t, s)=\alpha\left[\frac{1}{2}(t+s)-1\right]-\frac{1}{2}\left(t^{\alpha}+s^{\alpha}\right)+1, \quad(t, s) \in D
$$

We shall show that $\max \{h(t, s):(t, s) \in D\} \leqslant 0$. Since it is easy to see that $h$ does not have critical points in the interior of $D$, it suffices to show that $\max \{h(t, s):(t, s)$ $\in \partial D\} \leqslant 0$, where $\partial D$ is the boundary of $D$ given by $\partial D=D_{1} \cup D_{2} \cup D_{3}$, where

$$
\begin{aligned}
& D_{1}=\{(t, 1+\tau): 1-\tau \leqslant t \leqslant 1+\tau\} \\
& D_{2}=\{(1+\tau, s): 1-\tau \leqslant s \leqslant 1+\tau\} \\
& D_{3}=\{(t, s): t+s=2,1-\tau \leqslant t, s \leqslant 1+\tau\} .
\end{aligned}
$$

On $D_{1}$ we have

$$
\bar{h}(t) \equiv h(t, 1+\tau)=\alpha\left[\frac{1}{2}(t+1+\tau)-1\right]-\frac{1}{2}\left[t^{\alpha}+(1+\tau)^{\alpha}\right]+1, \quad 1-\tau \leqslant t \leqslant 1+\tau .
$$

Since $\vec{h}(t)=(\alpha / 2)\left(1-t^{\alpha-1}\right), \bar{h}$ is decreasing for $t \geqslant 1$ and increasing for $t \leqslant 1$. Hence

$$
\bar{h}(t) \leqslant \bar{h}(1)=\frac{1}{2}\left[(1+\alpha \tau)-(1+\tau)^{\alpha}\right] \leqslant 0 .
$$

Similarly, we can prove that $h(t, s) \leqslant 0$ on $D_{2}$ and $D_{3}$. Hence $\max \{h(t, s):(t, s)$ $\in \partial D\} \leqslant 0$ and the Claim has thus been proved. 
Now for $\|x\|=\|y\|=1,\|x \pm \tau y\| \leqslant 1+\tau$ and $\|x+\tau y\|+\|x-\tau y\| \geqslant 2$, it follows from the Claim and (2.2) that

$$
\frac{1}{2}(\|x+\tau y\|+\|x-\tau y\|)-1 \leqslant \frac{\lambda}{\alpha} \tau^{\alpha}, \quad x, y \in X,\|x\|=\|y\|=1 .
$$

This implies that

$$
\rho_{X}(\tau) \leqslant \frac{\lambda}{\alpha} \tau^{\alpha}, \quad 0 \leqslant \tau \leqslant 1
$$

and $X$ is $\alpha$-uniformly smooth.

We conclude this section by showing that in property $(U, \lambda, 1, \beta)$ one can assume $\beta=\alpha-1=0$.

TheOrem 2.3. Assume that a Banach space $X$ has $(U, \lambda, 1, \beta)$. Then $X$ also has $(U, \lambda, 1,0)$.

Proof: By the definition of property $(U, \lambda, 1, \beta)$, we see that the largest $\widetilde{\beta}$ such that property $(U, \lambda, 1, \widetilde{\beta})$ holds for $X$ is determined by

$$
2^{\tilde{\beta}}=\inf \left\{\frac{\|x+y\|+\lambda\|x-y\|}{\|x\|+\|y\|}: x, y \in X,\|x\|+\|y\| \neq 0\right\} .
$$

All we need to show is that the infimum in (2.3) equals 1 (and hence $\widetilde{\beta}=0=\alpha-1$ ). Indeed, if we rewrite the function on the right-hand side of (2.3) as

$$
1+\frac{\|x+y\|+\lambda\|x-y\|-(\|x\|+\|y\|)}{\|x\|+\|y\|}
$$

and observe (note that $\lambda \geqslant 1$ ) that

$$
\|x+y\|+\lambda\|x-y\| \geqslant\|x+y\|+\|x-y\| \geqslant\|x\|+\|y\|,
$$

we conclude that the infimum in (2.3) is attained at any point $(x, x)$ with $x \neq 0$ and that it does indeed equal 1.

\section{Property $(U, 1, \alpha, 1)$}

A Hilbert space has property $(U, 1,2,1)$. The following result shows that property $(U, 1, \alpha, 1)$ with $1<\alpha \leqslant 2$ characterises Hilbert spaces. Note that Clarkson's inequalities (1.8) show that if $L^{p}$ (or $l^{p}$ ) has property $(U, 1, p, 1)$ with $1<p \leqslant 2$, then $p=2$ and $L^{p}$ reduces to a Hilbert space. Below we extend this to the general case.

TheOrem 3.1. If a Banach space $X$ has property $(U, 1, \alpha, 1)$ for some $1<\alpha \leqslant 2$, then $X$ is a Hilbert space.

Proof: By property $(U, 1, \alpha, 1)$ we have

$$
\|x+y\|^{\alpha}+\|x-y\|^{\alpha} \geqslant 2\left(\|x\|^{\alpha}+\|y\|^{\alpha}\right), \quad x, y \in X .
$$


Taking $x=y$, we see that $2^{\alpha} \geqslant 4$ which implies $\alpha \geqslant 2$. Hence $\alpha=2$. So we can rewrite (3.1) as

$$
\|x+y\|^{2}+\|x-y\|^{2} \geqslant 2\left(\|x\|^{2}+\|y\|^{2}\right), \quad x, y \in X
$$

Setting $x=(u+v) / 2$ and $y=(u-v) / 2$ in (3.2), we obtain

$$
\|u+v\|^{2}+\|u-v\|^{2} \leqslant 2\left(\|u\|^{2}+\|v\|^{2}\right), \quad u, v \in X
$$

Taken together, the inequalities (3.2) and (3.3) are equivalent to the parallelogram identity:

$$
\|x+y\|^{2}+\|x-y\|^{2}=2\left(\|x\|^{2}+\|y\|^{2}\right), \quad x, y \in X
$$

Therefore $X$ is a Hilbert space.

\section{Dual Property}

In this section we introduce the dual property of $(U, \lambda, \alpha, \beta)$.

DEFinition 4.1: A Banach space $X$ is said to have property dual $(U, \lambda, \alpha, \beta)$, denoted by $\left(U^{*}, \lambda, \alpha, \beta\right)$, if, for some $\lambda>0, \alpha>1$ and $\beta>0$, there holds

$$
\|x+y\|^{\alpha}+\lambda\|x-y\|^{\alpha} \leqslant 2^{\beta}\left(\|x\|^{a}+\|y\|^{\alpha}\right), \quad x, y \in X .
$$

In analogy with Theorem 2.2 , we shall show that property $\left(U^{*}, \lambda, \alpha, \beta\right)$ is equivalent to $\alpha$-uniform convexity. But first recall that the modulus of convexity of $X$ is defined by

$$
\delta_{X}(\varepsilon)=\inf \left\{1-\frac{1}{2}\|x+y\|:\|x\|=\|y\|=1,\|x-y\|=\varepsilon\right\} .
$$

Recall also that $X$ is uniformly convex if $\delta_{X}(\varepsilon)>0$ for all $\varepsilon>0$ and for $1<p<\infty, X$ is p-uniformly convex if, for some constant $c>0$,

$$
\delta_{X}(\varepsilon) \geqslant c \varepsilon^{p}, \quad \varepsilon>0 .
$$

It is known that a Hilbert space and $l^{p}$ (or $L^{p}$ ) for $1<p \leqslant 2$ are 2-uniformly convex; while $l^{p}$ (or $L^{p}$ ) for $2 \leqslant p<\infty$ is $p$-uniformly convex (see [7]).

Clarkson's inequalities (1.8) show that as a uniformly convex Banach space, $L^{p}$ (or $\left.l^{p}\right)$ also has the dual property $\left(U^{*}, 1, p, p-1\right)$ for $2 \leqslant p<\infty$ and $\left(U^{*}, 1, p, 1\right)$ for $1<p<2$. Note that $L^{p}$ (or $l^{p}$ ) is both uniformly convex and uniformly smooth. Our purpose in this section is to extend these properties for $L^{p}$ (or $l^{p}$ ) to the more general class of $\alpha$-uniformly convex Banach spaces. To this end, we need an inequality characterisation of $p$-uniform convexity (see $[7,3]$ ).

Proposition 4.2. Let $X$ be a Banach space and let $1<p<\infty$ be a real number. Then $X$ is $p$-uniformly convex if and only if there exists a constant $c>0$ with the property:

$$
\|t x+(1-t) y\|^{p} \leqslant t\|x\|^{p}+(1-t)\|y\|^{p}-c W_{p}(t)\|x-y\|^{p}
$$


for all $t \in[0,1]$ and $x, y \in X$, where $W_{p}(t)=t^{p}(1-t)+t(1-t)^{p}$.

THEOREM 4.3. Let $X$ be a Banach space and let $1<\alpha<\infty$ be a real number. Then $X$ has property $\left(U^{*}, \lambda, \alpha, \alpha-1\right)$ if and only of $X$ is $\alpha$-uniformly convex.

Proof: Assume first that $X$ is $\alpha$-uniformly convex. From (4.2) it follows that

$$
\left\|\frac{x+y}{2}\right\|^{\alpha} \leqslant \frac{1}{2}\|x\|^{\alpha}+\frac{1}{2}\|y\|^{\alpha}-\frac{c}{2^{\alpha}}\|x-y\|^{\alpha}, \quad x, y \in X .
$$

This implies that $X$ has $\left(U^{*}, \lambda, \alpha, \alpha-1\right)$ with $\lambda=c$.

Conversely, assume that $X$ has $\left(U^{*}, \lambda, \alpha, \alpha-1\right)$. If $\|x\|=\|y\|=1$ and $\|x-y\|=\varepsilon$, we have by (4.1),

$$
\left\|\frac{x+y}{2}\right\|^{\alpha} \leqslant 1-\lambda\left(\frac{\varepsilon}{2}\right)^{\alpha} \text {. }
$$

This implies that

$$
\delta_{X}(\varepsilon) \geqslant 1-\left[1-\lambda\left(\frac{\varepsilon}{2}\right)^{\alpha}\right]^{1 / \alpha} \geqslant \frac{\lambda}{\alpha}\left(\frac{\varepsilon}{2}\right)^{\alpha}
$$

Hence $X$ is $\alpha$-uniformly convex.

Corollary 4.4. Let $X$ be a Banach space and let $\alpha>1$ be a real number. Then $X$ has property $(U, \lambda, \alpha, \alpha-1)$ for some $\lambda>0$ if and only if $X^{*}$ has property $\left(U^{*}, \lambda^{\prime}, \alpha^{\prime}, \alpha^{\prime}-1\right)$ for some $\lambda^{\prime}>0$, where $1 / \alpha+1 / a^{\prime}=1$.

ProOF: By Theorem 2.2, we see that $X$ has $(U, \lambda, \alpha, \alpha-1)$ if and only if $X$ is $\alpha$ uniformly smooth, which is equivalent to $X^{*}$ being $\alpha^{\prime}$-uniformly convex, which is in turn, by Theorem 4.3, equivalent to $X^{*}$ having property $\left(U^{*}, \lambda^{\prime}, \alpha^{\prime}, \alpha^{\prime}-1\right)$ for some $\lambda^{\prime}>0$. $\square$

\section{REFERENCES}

[1] B. Beauzamy, Introduction to Banach spaces and their geometry, North Holland Mathematical Library 42 (North-Holland, New York, 1982).

[2] J.A. Clarkson, 'Uniformly convex spaces', Trans. Amer. Math. Soc. 40 (1936), 396-414.

[3] T.H. Kim and H.K. Xu, 'Some Hilbert space characterizations and Banach space inequalities', Math. Inequal. Appl. 1 (1998), 113-121.

[4] J. Lindenstrauss and L. Tzafriri, Classical Banach Spaces I (Springer-Verlag, Berlin, Heidelberg, New York, 1979).

[5] J. Schu, 'On a theorem of C.E. Chidume concerning the iterative approximation of fixed points', Math. Nachr. 153 (1991), 313-319.

[6] Yu. V. Trubnikov, 'The Hanner inequality and the convergence of iterative processes', (Russian)., Izv. Vyssh. Uchebn. Zaved. Mat. 84 (1987), 57-64.English translation: Soviet Math. (Iz. VUZ) 31 (1987) 74-83 MR 89a:46029.

[7] H.K. Xu, 'Inequalities in Banach spaces with applications', Nonlinear Anal. 16 (1991), 1127-1136. 
Department of Mathematics The Technion-Israel Institute of Technology 32000 Haifa

Israel

e-mail: sreich@tx.technion.ac.il
Department of Mathematics University of Durban-Westville Private Bag X54001

Durban 4000

South Africa

e-mail: hkxu@pixie.udw.ac.za 